\title{
SATISFACCIÓN DEL USUARIO DE LOS SERVICIOS DE CONSULTA EXTERNA DE PEDIATRÍA DEL HOSPITAL GUILLERMO ALMENARA IRIGOYEN, LIMA, PERÚ, 2007
}

\author{
Magdieli TITO HERMITAÑO ${ }^{1}$, Roussel DÁVILA VILLAVICENCIO ${ }^{2}$
}

\begin{abstract}
RESUMEN
Objetivo: Determinar el grado de satisfacción de los usuarios de los servicios de consulta externa de pediatría del Hospital Guillermo Almenara Irigoyen e identificar posibles mejoras. Metodología: El estudio es transversal descriptivo, para el cual se empleó un cuestionario de satisfacción, el mismo que fue administrado a las madres que acudieron en búsqueda de atención para sus hijos en los consultorios externos de pediatría durante la primera semana del mes de julio del 2007. La muestra estuvo constituida por 184 madres de una población de 355. Resultados: De las 184 madres de familia encuestadas que acudieron al consultorio de pediatría $69 \%$ conoce el nombre del pediatra y en un $47 \%$ conoce el nombre del enfermero o enfermera. El $75 \%$ manifiesta que el tiempo de atención es correcto, el $87 \%$ indica que el personal casi siempre fue amable y el $83 \%$ que casi siempre el personal se interesó por prestarle ayuda. En cuanto a las condiciones físicas, en un promedio mayor al $70 \%$ las madres percibieron que era como lo esperaban. En la escala numérica del 1 al 10 en la que el paciente señaló el grado de recomendación de servicio el 66.3\%, asignó 7 y 8 . Conclusión: En general el servicio de pediatría es recomendable desde el punto de vista de la experiencia de satisfacción de las madres encuestadas en una puntuación media de 7 en la escala de 0 a 10, debiendo mejorar la rapidez en el tiempo de espera, puntualidad en la atención y optimizar el trato amable en todo el proceso de atención.
\end{abstract}

Palabras clave: Satisfacción, usuario, consulta pediatría.

\begin{abstract}
Objective: To determine the users' satisfaction degree in the outpatient Pediatric Services at the Guillermo Almenara Irigoyen General Hospital and identify possible improvements. Methodology: This is a cross-sectional descriptive study, for which it was used a satisfaction questionnaire that was administered to mothers who came looking for health care for their children in the pediatric outpatient clinic during the first week of July 2007. The sample consisted of 184 mothers in a population of 355. Results: From the 184 mothers surveyed who visited the pediatric clinic $69 \%$ knew the doctor's name and $47 \%$ knew the nurse's name. $75 \%$ said that service time is right, $87 \%$ indicated that the staff was friendly and $83 \%$ said that most of the time the staff showed interest in helping them. As for the physical conditions of the facilities, an average of more than $70 \%$ of mothers perceived that it was as they had expected. In the numerical scale from 1 to 10 in which the patient had to rate the recommendation for this service, $66.3 \%$ marked 7 and 8 . Conclusions: In general pediatrics service is recommended from the standpoint of the satisfaction experience of mothers surveyed in an average score of 7 on a scale from 0 to 10 . The recommendation would be to improve the time in the waiting process, prompt attention and optimize the friendliness in the whole process of care.
\end{abstract}

Key Words: Satisfaction, user, pediatric service.

\footnotetext{
${ }^{1}$ Lic. en Enfermería, Especialista en Administración y gestión en Enfermería, Docente de la Facultad de Ciencias de la Salud de la Universidad Peruana Unión.

${ }^{2}$ Lic. en Enfermería, Especialista en Administración y gestión en Enfermería, Docente de la Facultad de Ciencias de la Salud de la Universidad Peruana Unión.
} 


\section{INTRODUCCIÓN}

El concepto de calidad aplicado a la atención médica hace referencia a la capacidad que con distinto grado, puede tener una organización o un acto concreto de asistencia sanitaria para satisfacer las necesidades de los consumidores de servicios de salud.

La definición que la OMS da a calidad es "alto nivel de excelencia profesional usando eficientemente los recursos con un mínimo de riesgos para el paciente, para lograr un alto grado de satisfacción por parte de éste y produciendo un impacto final positivo en salud".

Es este sentido la satisfacción del usuario es el aspecto fundamental de medición de la calidad e implica una experiencia racional o cognoscitiva, derivada de la comparación entre las expectativas y el comportamiento del producto o servicio, está subordinada a numerosos factores como las expectativas, valores morales, culturales, necesidades personales y la propia organización sanitaria. Estos elementos condicionan que la satisfacción sea diferente para distintas personas y para la misma persona en diferentes circunstancias.

El objetivo de los servicios sanitarios es satisfacer las necesidades de sus usuarios, y el análisis de la satisfacción es un instrumento de medida de la calidad de atención. Esta medición permite tener en cuenta las necesidades y expectativas del usuario a la hora de tomar decisiones clínicas (Relman, 1988).

La importancia de la medición de la satisfacción del usuario ha sido demostrada en diferentes estudios por su estrecha relación con elementos como el cumplimiento en el tratamiento y la mejora en el estado de salud, tal como lo demuestran investigadores como Yancy (2007), McKinley (1997) y Ross (1995). La calidad de atención en la consulta externa debe ser uno de los objetivos importantes del sistema sanitario nacional, debido a que éste registra siempre un alto volumen de pacientes produciendo largas colas de espera que generan insatisfacción (Nuñez, 2002).

Los estudios sobre satisfacción de usuarios en consultorios externos son escasos y mucho más en servicios de pediatría, sin embargo en los servicios hospitalarios es frecuente este tipo de evaluaciones mediante encuestas. Empero, es necesario considerar que las expectativas de los pacientes hospitalizados son diferentes a los ambulatorios por las característica mismas de la atención requerida por lo que es importante conocerlas. Esta situación genera una oportunidad para la ejecución de investigaciones en una realidad específica como es el caso del Hospital Guillermo Almenara Irigoyen que cuenta con servicios ambulatorios dentro de ellos de pediatría, donde no se han identificado claramente las expectativas del usuario a diferencia de los servicios de hospitalización donde esta medición se aplica periódicamente con instrumentos que la misma institución ha implementado.

En el presente estudio se aplicó una encuesta con el objetivo de determinar el grado la satisfacción de las madres con la atención que reciben sus menores hijos en el consultorio externo de pediatría, y de manera específica: determinar el grado de satisfacción con la atención recibida por el personal, determinar el grado de satisfacción con las condiciones físicas, establecer el grado de satisfacción con la información recibida e identificar posibles problemas desde la expectativa del usuario.

\section{MATERIAL Y MÉTODOS}

El estudio fue transversal descriptivo, se realizó en la primera semana de julio del 2007, y se encuestaron a una muestra de 184 madres de familia que acudieron al consultorio de pediatría acompañando a sus menores hijos. Esta muestra fue obtenida mediante cálculo de muestreo considerando a una población semanal promedio de 350 usuarios. La encuesta fue entregada a las madres después de salir de los dos consultorios pediátricos, mediante selección sistemática (1 por cada dos atendidos), y fue contestada por escrito en forma anónima y voluntaria.

El cuestionario empleado es una adaptación del modelo empleado por la Asociación de Urólogos de Columbia de los Estados Unidos y por el Hospital Universitario Virgen de la Arrixaca, Murcia (España).

El cuestionario contó con preguntas para los indicadores demográficos, mediante una escala de likert sobre la atención recibida, las condiciones físicas y la información recibida por el paciente. Adicionalmente se incluyó preguntas dicotómicas para identificar problemas y una pregunta abierta para identificar el problema específico.

La posible recomendación del servicio a algún familiar o amigo fue analizada según una escala visual de 1 al 10 (desde nunca, hasta sin dudarlo). Finalmente se consideró una pregunta abierta para solicitar sugerencias. En total el cuestionario contuvo 24 preguntas.

El proceso de recojo de datos así como el análisis de los mismos estuvo a cargo del equipo investigador, valiéndose para este último proceso el empleo del software estadístico SPSS en su versión 11.5. 


\section{RESULTADOS}

Los resultados de la encuesta revelan que la media de edad de las madres encuestadas fue de 31 años y la media de edad de los niños atendidos fue de 3 años.

Tabla 1. Nivel sociocultural de las madres acompañantes de niños atendidos en los servicios de consulta externa de pediatría del Hospital Guillermo Almenara Irigoyen, Lima, Perú, 2007.

\begin{tabular}{|l|cc|}
\hline Nivel de estudios & $\mathrm{N}^{0}$ Madres & $\%$ \\
\hline Sin estudios & 4 & 2,2 \\
Primaria & 34 & 18,5 \\
Secundaria & 113 & 61,4 \\
Superior & 33 & 17,9 \\
\hline
\end{tabular}

Fuente: instrumento aplicado a las madres acompañantes de servicios de Pediatría del HNGAI, 2007.

La distribución de las madres encuestadas según la variable nivel de estudios indica que el mayor porcentaje lo representa el grupo de estudios secundarios con un $61.4 \%$ y con un $2.2 \%$ el grupo sin ningún nivel de instrucción tal como se muestra en la (Tabla $\mathrm{N}^{\mathrm{o}} 1$ ).

Por otro lado, sólo 15 madres acompañantes (8.2\%) acudían por primera vez al consultorio de pediatría y 169 (91.1\%) ya lo habían hecho anteriormente. Asimismo, 20 madres habían tenido la experiencia de recibir atención pediátrica de sus hijos en otro hospital (10.9\%) y 164 de ellas no habían tenido dicha experiencia $(89.1 \%)$.

Tabla 2. Satisfacción con la atención recibida en los servicios de consulta externa de pediatría del Hospital Guillermo Almenara Irigoyen, Lima, Perú, 2007

\begin{tabular}{|c|c|c|c|}
\hline Nivel de estudios & & $\mathrm{N}^{0}$ de madres & $\%$ \\
\hline \multirow{2}{*}{ Nombre del pediatra } & Lo conoce & 127 & 69,0 \\
\hline & No lo conoce & 57 & 31,0 \\
\hline \multirow{2}{*}{ Nombre del enfermero } & Lo conoce & 87 & 47,3 \\
\hline & No lo conoce & 97 & 52,7 \\
\hline \multirow{5}{*}{ Tiempo de atención } & Muy escaso & - & - \\
\hline & Escaso & 46 & 25,0 \\
\hline & Correcto & 138 & 75,0 \\
\hline & Excesivo & - & - \\
\hline & Muy excesivo & - & - \\
\hline \multirow{4}{*}{$\begin{array}{l}\text { El personal de salud fue } \\
\text { amable }\end{array}$} & Nunca & - & - \\
\hline & Pocas veces & 14 & 7,6 \\
\hline & Casi siempre & 160 & 87,0 \\
\hline & Siempre & 10 & 5,0 \\
\hline \multirow{4}{*}{ El personal de salud le ayudó } & Nunca & - & - \\
\hline & Pocas veces & 30 & 16,3 \\
\hline & Casi siempre & 154 & 83,7 \\
\hline & Siempre & - & - \\
\hline
\end{tabular}

Fuente: instrumento aplicado a las madres acompañantes de servicios de Pediatría del HNGAI, 2007.

La tabla 2 muestra que un $69 \%$ de las madres acompañantes conocía el nombre del médico y solo un $47 \%$ conocía el nombre del enfermero o enfermera que asistiría la atención. En cuanto al tiempo de atención dedicado en la consulta para el niño, un 75\% y lo consideraron "correcto", y "escaso" sólo el 25\%. Respecto a la amabilidad del personal en el trato, un 92.4\% opinó que está presente "casi siempre" o "siempre". Las encuestadas manifestaron también en un $83.7 \%$ que "casi siempre" sintieron que el personal hizo lo posible por ayudarle cuando lo necesitaron y un $16,3 \%$ opinó que esto sucedió pocas veces. 
Tabla 3. Satisfacción con las condiciones físicas de los servicios de consulta externa de pediatría del Hospital Guillermo Almenara Irigoyen, Lima, Perú, 2007

\begin{tabular}{|c|c|c|c|}
\hline Nivel de estudios & & $\mathrm{N}^{\mathrm{o}}$ de madres & $\%$ \\
\hline \multirow{5}{*}{ Temperatura de la sala } & Mucho peor & 4 & 2,2 \\
\hline & Peor & 43 & 23,4 \\
\hline & Como lo esperaba & 137 & 74,5 \\
\hline & Mejor & - & - \\
\hline & Mucho mejor & - & - \\
\hline \multirow{5}{*}{ Ruido en sala de espera } & Mucho peor & - & - \\
\hline & Peor & 33 & 17,9 \\
\hline & Como lo esperaba & 147 & 79,9 \\
\hline & Mejor & 4 & 2,2 \\
\hline & Mucho Mejor & - & - \\
\hline \multirow{5}{*}{ Limpieza de la sala de consulta } & Mucho peor & - & - \\
\hline & Peor & 27 & 14,7 \\
\hline & Como lo esperaba & 157 & 85,3 \\
\hline & Mejor & - & - \\
\hline & Mucho mejor & - & - \\
\hline
\end{tabular}

Fuente: instrumento aplicado a las madres acompañantes de servicios de Pediatría del HNGAI, 2007.

En la tabla 3, en relación a las condiciones físicas que incluye la temperatura de la sala de consulta, el ruido en la sala de espera y la limpieza en el consultorio, la mayoría de las encuestadas mostró satisfacción porque consideran que fue "como lo esperaban" (74\%, 79\% y 85\% respectivamente).

Tabla 4. Satisfacción con la información recibida en los servicios de consulta externa de pediatría del Hospital Guillermo Almenara Irigoyen, Lima, Perú, 2007

\begin{tabular}{|c|c|c|c|}
\hline Nivel de estudios & & $\mathrm{N}^{\circ}$ de madres & $\%$ \\
\hline \multirow{5}{*}{$\begin{array}{l}\text { Información general de la } \\
\text { enfermedad }\end{array}$} & Nunca & - & - \\
\hline & Pocas veces & - & - \\
\hline & Casi siempre & 23 & 12,5 \\
\hline & Siempre & 161 & 87,5 \\
\hline & No procede & - & - \\
\hline \multirow{5}{*}{$\begin{array}{l}\text { Pruebas diagnósticas } \\
\text { realizadas }\end{array}$} & Nunca & - & - \\
\hline & Pocas veces & 9 & 4,9 \\
\hline & Casi siempre & 39 & 21,2 \\
\hline & Siempre & 112 & 60,9 \\
\hline & No procede & 24 & 13,0 \\
\hline \multirow{5}{*}{$\begin{array}{l}\text { Evolución de los síntomas } \\
\text { tras el tratamiento }\end{array}$} & Nunca & - & - \\
\hline & Pocas veces & - & - \\
\hline & Casi siempre & 38 & 20,7 \\
\hline & Siempre & 122 & 66,3 \\
\hline & No procede & 24 & 13,0 \\
\hline \multirow{5}{*}{$\begin{array}{l}\text { Riesgos del diagnóstico y } \\
\text { el tratamiento }\end{array}$} & Nunca & - & - \\
\hline & Pocas veces & 15 & 8,2 \\
\hline & Casi siempre & 29 & 15,8 \\
\hline & Siempre & 121 & 65,8 \\
\hline & No procede & 19 & 10,3 \\
\hline
\end{tabular}

En la tabla 4 se observa que el análisis de las preguntas referidas a la información recibida muestra que un 100\% de las madres manifestó que recibieron "siempre" (87.5\%) y "casi siempre" (12.5\%) información general de la enfermedad de niño. Un $82.1 \%$ opinó que recibieron "siempre" $(60.9 \%)$ y "casi siempre" $(21,2 \%)$ información referente a las pruebas diagnósticas. En cuanto a las información relacionada con la evolución de síntomas tras el tratamiento un $87 \%$ indicó que "siempre" (66.3\%) y "casi siempre" (20.7\%) la recibió. Un 81\% manifestó que "siempre" $(65.8 \%)$ y "casi siempre" $(15.8 \%)$ fue informado respecto al diagnóstico y tratamiento. 
Las preguntas relacionadas a la existencia o no de problemas en determinados aspectos de la atención recibida muestran algunas cifras tales como: 13 madres indicaron problemas con la acogida o trato en el servicio (7,1\%), 4 de ellas indicó haber tenido algún problema con el diagnóstico de la enfermedad principalmente por la variación de mismo $(2.2 \%)$ y finalmente 17 madres refirieron haber tenido algún problema con la información recibida $(9,2 \%)$.

Respecto a la escala numérica de 1 al 10 (nulo, sin dudarlo) en la que la madre señaló el grado de recomendación del servicio a un familiar o amigo, en caso éste lo necesite, encontramos que la media de la puntuación asignada corresponde a 7. Cabe decir que el servicio se consideró "recomendable" cuando los pacientes asignaron un puntaje de 7 a más, y "no recomendable o dudoso" cuando asignaron un puntaje menor de 7. En este sentido se observó que un $66.3 \%$ de las madres asignó un puntaje de 7 a más, lo que indica que el servicio es recomendable en esta proporción. Es necesario mencionar que no existieron puntuaciones asignadas menores de 5 .

Del total de encuestadas, el $76.6 \%$ registró recomendaciones para el servicio: el 35.3\% recomendó mejorar la puntualidad en la atención y disminución del tiempo de espera, un $28.8 \%$ manifestó sugerencias para mejorar el trato amable y gentil del personal y vigilantes. La recomendación referente a la ampliación de citas fue considerada por un $5.4 \%$, la mejora de la información del personal de salud incluyendo a los vigilantes $4.9 \% \mathrm{y}$, finalmente, la ampliación de la planta física del consultorio fue mencionada por un $2.2 \%$ de las encuestadas.

\section{DISCUSIÓN}

Los clientes se están transformando cada vez más en compradores conscientes de la calidad y exigen que sus proveedores cumplan con los más altos requisitos de calidad, tanto del servicio como del producto.

Si bien es cierto que en la actualidad han evolucionado una serie de estándares de acreditación, como por ejemplo el modelo EFQM y el ISO 9001:2000 que difieren en cuanto a su contenido, pero que sin embargo tienen grandes similitudes, siendo una de ellas la orientación hacia el cliente destacando la importancia de la información contrastada sobre las preferencias y tendencias de la opinión de los distintos tipos de clientes.

El modelo EFQM señala que es básico que el sistema de calidad de toda organización recoja información objetiva sobre las preferencias y expectativas para enfocar los servicios que brinda dicha institución a las ne- cesidades cambiantes de los clientes, es decir en el caso de los sistemas de salud de los pacientes o usuarios.

En el sector salud, desde hace algunos años, se viene usando diferentes métodos para identificar los aspectos más importantes para la mejora de los servicios. Así por ejemplo Hall y Dorman (1988) muestran que en las encuestas empleadas para los estudios se le daba mayor relevancia a la "humanización" (65\% de los casos), a la cantidad de información que se brindaba al paciente (50\%) y a la competencia percibida de los profesionales $(43 \%)$. Posteriormente otros autores como Mckinley (1997) y Nathorst-Boos (2001), mediante el uso de otros enfoques metodológicos, han identificado como factores importantes para determinar el nivel de satisfacción las condiciones del entorno físico, la accesibilidad (no sólo la física), la comunicación entre el médico y el paciente, los cuidados de enfermería que se facilitan, la competencia profesional, la cortesía y amabilidad en el trato y el resultado alcanzado.

En el ámbito local y nacional existen numerosos estudios de satisfacción de pacientes básicamente en el área de los servicios de hospitalización, sin embargo, no se ha podido identificar investigaciones que valoren la satisfacción de los pacientes que acuden a consultas de especialidades y particularmente en el caso de los consultorios de pediatría de la seguridad social del Perú. En este sentido, el presente estudio constituye un valioso aporte considerando que muestra una visión global de la atención recibida por el paciente de pediatría mediante la opinión de la madre acompañante.

Una característica sociodemográfica importante recogida en el estudio fue el grado de instrucción, encontrando que el $61.4 \%$ tiene educación secundaria y un $17 \%$ educación superior. Este aspecto es de consideración importante tomando como referencia a Vicuña (2002), quien en un investigación realizada en el Hospital Hipólito Unanue en Lima, Perú, determinó que el mayor grado de instrucción influye inversamente en la satisfacción con el servicio, es decir los de mayor grado de instrucción tienden a ser más exigentes con los servicios que reciben durante la atención. En este sentido, se entiende que los datos reportados en el presente estudio en su mayoría reportan datos producto de una alta exigencia.

Según Relman (1988) y Ross (1995), el factor denominado “calidad subjetiva” corresponde a los aspectos relacionados con la amabilidad, la empatía o si al paciente le pareció adecuado el tiempo de consulta que se le dedicó. Además, el conocimiento del nombre del médico y concretamente que éste se haya presentado constituyen un hecho fundamental para mejorar la receptividad del paciente mejorando su conducta terapéutica. 
En este sentido, el hecho de que un $75 \%$ considerara al tiempo de la consulta como correcto, el 92.4\% que el personal fue amable en su trato "casi siempre" o "siempre" y que un $83.7 \%$ indique que casi "siempre" sintieron que el personal hizo lo posible por ayudarle cuando lo necesitaron, muestra las condiciones favorables del servicio. Cabe indicar que las recomendaciones de un grupo importante de las madres para mejorar la atención reitera el trato amable y gentil de parte del personal asistencial y de los vigilantes (28.8\%).

Sin embargo, el estudio también muestra que un 69\% conoce el nombre del pediatra y sólo un $47,3 \%$ conoce el nombre de la enfermera, cifras que deben ser mejoradas considerando el impacto que producen en el paciente tal como se manifestó en el párrafo anterior.

Por otro lado, la "calidad objetiva" incluye las opiniones referentes al estado físico de la consulta, el tiempo de espera, la puntualidad de las consultas o la información dada por el médico. En este sentido, los datos encontrados son favorables, sin embargo es necesario tomar atención en el hecho de que un 25.6\% indica que la temperatura del ambiente de la sala estuvo "peor" y "mucho peor" de los que esperaban, un 17.9\% opinó que el ruido en la sala de espera fue "peor" de lo que esperaban y $14.7 \%$ opinó que la limpieza de la sala de consulta estuvo "peor” de lo esperado. Esta situación muestra oportunidades de mejora y optimización en la prestación de servicios. Además se deberá considerar las recomendaciones más importantes manifestadas por las madres encuestadas en este rubro como son: mejorar la puntualidad en la atención y el tiempo de espera para la consulta $(35.3 \%)$ y la ampliación de citas $(5.4 \%)$.

Los aspectos relacionados a la satisfacción con la información recibida muestran cifras muy favorables considerando que superan el $80 \%$. Aunque los porcentajes en el que "pocas veces" se brindo información es bajo, es necesario remarcarlo para su optimización, es así que un $8.2 \%$ opinó que "pocas veces" se les informó sobre las pruebas diagnósticas y sólo un 4,9 refiere que la información sobre los riesgos del diagnóstico y tratamiento fue dado “pocas veces”. Incidir en estos aspectos a mejorar es importante; Según Coulter (1998), están relacionados con la autonomía como base de la práctica clínica y constituye un aspecto importante en la interactuación entre el médico y el paciente.

\section{CONCLUSIONES}

1. La mayoría de las madres acompañantes de niños atendidos en consultorios de pediatría $(66.3 \%)$ recomendarían a un familiar o amigo producto de su satisfacción con el servicio.

2. La mayoría de las madres muestran satisfacción con la tención recibida sin embargo se debe mejorar la disposición del personal para prestar ayuda al paciente y éste lo perciba.

3. Un porcentaje importante de las madres no conocen el nombre del médico (31\%) ni de la enfermera $(52.7 \%)$, factor que es importante superar para ejercer un mayor impacto en la receptividad y conducta terapéutica del paciente o acompañante.

4. La mayoría de las madres muestran satisfacción con las condiciones físicas, sin embargo existen porcentajes importantes de madres que muestran su insatisfacción con la temperatura de la sala (25.6), ruido de la sala de espera (17.9\%) y la limpieza de la sala $(14.7 \%)$. Estos datos indican oportunidades de mejora.

5. La mayoría de las madres muestran satisfacción con la información recibida respecto a la información de la enfermedad, pruebas diagnósticas realizadas, evolución de los síntomas tras el tratamiento y riesgos del diagnóstico y tratamiento.

6. La mayoría de las madres recomendaron mejorar los siguientes aspectos: la puntualidad en la atención y el tiempo de espera para la consulta (35.3\%), amabilidad y gentileza del personal de salud y vigilantes $(28.8 \%)$. 


\section{REFERENCIAS BIBLIOGRÁFICAS}

1. AENOR. ISO 9000. Guía y comentarios. 3. ${ }^{\mathrm{a}}$ ed. Madrid: AENOR,2001. Benites L. y Ortellano A. Conocimientos, actitudes y prácticas acerca de los niños hospitalizados. Rev. Pediatría. España. Vol. 30, No 2; 2003. disponible en: http://www.spp.org. py/revistas/ed_2003/ninospers_jul_dic_2003.htm

2. Coulter A. Evidence based patient information. BMJ; 1998.

3. Hall J, Dornan M. Meta-analysis of satisfaction with medical care: description of research domain and analysis of overall satisfaction levels. Soc Scie Med; 1988.

4. McKinley R.K, Manku-Scott T, Hastings A.M, French D.P, Baker R.Reliability and validity of a new measure of patient satisfaction with out of hours primary medical care in the United Kingdom: development of a patient questionnaire. BMJ; 1997.

5. Nathorst-Böös J, Munck IME, Eckerlund I, EkfeldtSanberg C. An evaluation of the QSP and the QPP: two methods for measuring patient satisfaction. Int J Quality Health Care; 2001.
6. Núñez Díaz S, Martín-Martín AF, Rodríguez Palmero I, González Hernández J, Antolín Arias J, Hernández González R et. al. Identificación de problemas de calidad en la información clínica a usuarios. Rev Clin Esp; 2002.

7. Relman A.S. Assesment and Accountability. The third revolution in medical care. $\mathrm{N}$ Engl J Med; 1988.

8. Ross CK, Steward CA, Sinacore JM. A comparative study of seven measures of patient satisfaction. Med Care; 1995.

9. Vicuña M. Nivel de satisfacción y disfunciones percibidas sobre la calidad de atención de los servicios obstétricos. Anales de la Facultad de Medicina de la Universidad Mayor de San Marcos, Lima. Vol. 16, $\mathrm{N}^{\mathrm{o}}$ 01; 2002.

10. Yancy WS, Macpherson DS, Hanusa B, Switzer GE, Arnold RM, Buranosky RA et al. Patient satisfaction in resident and attending ambulatory care clinics. J Gen Intern Med; 2001. 